Conclusion: This is the largest study ever conducted to evaluate the impact of IA on male fertility. We demonstrated that men diagnosed with IA before and during their reproductive years have a lower fertility rate than those men diagnosed with IA after their reproductive years. Multiple mechanisms (biological and non-biological) can be responsible for this association. More research is needed to identify the causes of these lower fertility rates in men with IA.

REFERENCES:

[1] Perez-Garcia LF, Te Winkel B, Carrizales JP, Bramer W, Vorstenbosch S, van Puijenbroek E, et al. Sexual function and reproduction can be impaired in men with rheumatic diseases: A systematic review. Semin Arthritis Rheum. 2020;50(3):557-73

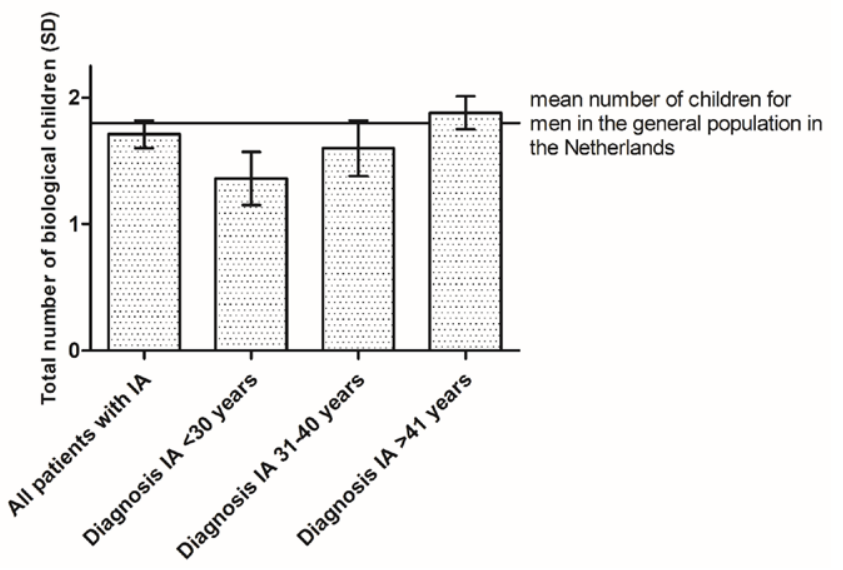

Figure 1. Total number of biological children (mean and SD) per group.

Acknowledgements: The authors would like to acknowledge Ron Buijs, data manager of the Department of Rheumatology of the Erasmus MC, for his technical support with regards to data collection.

Disclosure of Interests: Luis Fernando Perez-Garcia Consultant of: Galapagos, Esther Röder: None declared, Robbert Goekoop: None declared, Johanna Hazes: None declared, Marc R Kok Consultant of: Novartis, Grant/research support from: Novartis, Petra Kok: None declared, Hieronymus TW Smeele: None declared, Ilja Tchetverikov: None declared, J.H. van der Kaap: None declared, Annette van der Helm - van Mil: None declared, Bouwe Krijthe: None declared, Radboud Dolhain Speakers bureau: UCB, Roche, Abbvie, Genzyme, Novartis, Consultant of: Galapagos, Grant/research support from: UCB DOI: 10.1136/annrheumdis-2021-eular.639

\section{OP0213 PATIENTS WITH RHEUMATOID ARTHRITIS AND COMPARATORS IN A LARGE NATIONWIDE US STUDY}

H. J. Dykhoff ${ }^{1}$, E. Myasoedova ${ }^{2,3}$, M. Peterson ${ }^{2}$, J. M. Davis III², V. Kronzer², C. Coffey ${ }^{2}$, T. Gunderson ${ }^{3}$, C. S. Crowson ${ }^{2,3} .{ }^{1}$ Mayo Clinic, Robert D. and Patricia E. Kern Center for the Science of Health Care Delivery, Rochester, United States of America; ${ }^{2}$ Mayo Clinic, Division of Rheumatology, Rochester, United States of America; ${ }^{3}$ Mayo Clinic, Department of Quantitative Health Sciences, Rochester, United States of America

Background: Patients with rheumatoid arthritis (RA) have an increased burden of multimorbidity. Although many comorbidities vary by sex, sex differences in multimorbidity among individuals with RA have not been examined.

Objectives: We aimed to compare multimorbidity between women and men with RA and comparators without RA.

Methods: We used a large longitudinal, real-world data warehouse with de-identified administrative claims for commercial and Medicare Advantage enrollees, to identify cases of RA and matched controls. Cases were defined as patients aged $\geq 18$ years with $\geq 2$ diagnoses of RA in January 1, 2010 - June 30, 2019 and $\geq 1$ prescription fill for methotrexate in the year after the first RA diagnosis. Controls were persons without RA matched 1:1 to RA cases on age, sex, census region, calendar year of index date (corresponding to the date of second diagnosis code for RA), and length of prior medical/pharmacy coverage. Race was classified as non-Hispanic White (White), non-Hispanic Black (Black), Asian, Hispanic, or other/unknown, based on self-report or derived rule sets. Multimorbidity (2 or more comorbidities) was defined using 25 chronic comorbidities from a combination of the Charlson and Elixhauser Comorbidity Indices assessed during the year prior to index date. Rheumatic comorbidities were not included. Logistic regression models were used to estimate odds ratios (OR) with $95 \%$ confidence intervals $(\mathrm{Cl})$.

Results: The study included 16,363 cases with RA and 16,363 matched non-RA comparators (mean age 58.2 years, $70.7 \%$ female for both cohorts). In both cohorts, women were slightly younger (mean age 57.5 vs. 59.8 years). Among RA patients women were more racially/ethnically diverse than men, with $72 \%$ of women $(78 \%$ men) being White, 12\% (10\%) Hispanic, 11\% (7\%) Black, 3\% (3\%) Asian, and 3\% (3\%) other/unknown. Racial/ethnic diversity was similar among non-RA women and men with 74\% women (75\% men) being White, 9\% (9\%) Hispanic, 10\% (8\%) Black $4 \%(4 \%)$ Asian, and 3\% (4\%) other/unknown. Patients with RA had more multimorbidity than non-RA subjects ( $51.3 \%$ vs $44.8 \%)$. Observed rates of multimorbidity were similar in women and men with RA (51.6\% vs $50.5 \%, \mathrm{p}=0.18)$, but among non-RA patients, women had lower observed rates of multimorbidity than men $(43.7 \%$ vs $47.4 \%, p<0.0001$ ). However, following adjustment for age, race/ethnicity, and geographic region, multimorbidity among $\mathrm{RA}$ patients was greater in women versus men (OR: 1.19; 95\% Cl: 1.11-1.28) and similar among non-RA women and men (OR: 0.97 for females; $95 \% \mathrm{Cl}$ : 0.90-1.05). Examination of individual comorbidities showed that women with RA had more depression, hypothyroidism, diabetes mellitus, and chronic pulmonary disease compared to men with RA and women without RA.

Conclusion: This large nationwide study showed increased occurrence of multimorbidity in women with RA compared to men with RA, while women and men without RA had similar levels of multimorbidity following adjustment for age, race/ ethnicity, and geographic region. The underlying mechanisms for these sex differences require further investigation.

Disclosure of Interests: Hayley J. Dykhoff: None declared, Elena Myasoedova: None declared, Madeline Peterson: None declared, John M Davis III Grant research support from: Research grant from Pfizer, Vanessa Kronzer: None declared, Caitrin Coffey: None declared, Tina Gunderson: None declared, Cynthia S. Crowson: None declared

DOI: 10.1136/annrheumdis-2021-eular.1625

OP0214 IMPACT OF A MULTI-MORBIDITY SCREENING
AND PREVENTION PROGRAM IN CHRONIC INFLAMMATORY RHEUMATIC DISEASES ONTHE ONE-YEAR HOSPITALIZATION RATE BASED ON AN ANALYSIS OF THE FRENCH NATIONAL HEALTH DATABASE

G. Decarriere', J. Pastor ${ }^{2}$, D. Demoulin², G. Mouterde' ${ }^{1}$, C. Lukas ${ }^{1}$, B. Combe ${ }^{1}$ G. Mercier ${ }^{2}$, J. Morel ${ }^{1}$, C. Daien ${ }^{1} .{ }^{1} \mathrm{CHU}$, Rheumatology, Montpellier, France; ${ }^{2} \mathrm{CHU}$, Medical Information, Montpellier, France

Background: A screening program for multimorbidities started in 2014 at the Montpellier University Hospital for primary prevention in patients with chronic inflammatory rheumatic diseases (IRD).

Objectives: The objective of this work was to assess the impact of this program on morbidity by comparing the hospitalization rate of those patients in the year following the screening to the one of patients with IRD who did not benefit from this program. Methods: Patients with IRD who benefit from the screening program in 2015, 2016 and 2017 were identified in the French national health database PMSI and matched to 3 controls living in the same area on age, sex, type of IRD, use of intravenous (IV) biologic (b) DMARDs and index date. The exclusion criteria were subjects in secondary prevention identified as history of myocardial infarction in the previous 5 years or use of antiplatelet therapy. The primary outcome was the rate of all-cause hospitalization in the following year. The secondary endpoints were hospitalizations for another reason than IRD ("non-IRD") including those for cardiovascular [CV] events and major fractures. Hospitalization rates were compared between the two groups in the year after screening (or index date) and also between the year preceding screening and the year after for each group. Univariate and multivariate odds ratios (CI95\%) were calculated, taking into account the medical history (hypertension, diabetes, heart failure, CV disease, COPD, major fractures in the 5 years preceding the index date) and hospitalizations in the previous year.

Results: 486 patients were identified and matched with 1458 controls. $67.08 \%$ had rheumatoid arthritis and $21.81 \%$ spondyloarthritis; $7 \%$ of them had IV bDMARDs. Unscreened patients had more hypertension (19\% vs $10.1 \%$ ), diabetes ( $9 \%$ vs $4.9 \%)$, heart failure $(2.3 \%$ vs $0.4 \%)$ and "non-IRD" hospitalizations $(78.5 \%$ vs $72.2 \%)$ in the 5 years preceding the index date. In the year following the index date, the percentages of "all causes" and "non-IRD" hospitalizations were significantly higher in non-screened than in screened patients $(n=1944$, $64.8 \%$ versus $51 \%$, Chi2 test, $p<0.001$; and $47.1 \%$ versus $37.9 \%, p<0.001$ respectively). 17 (1.17\%) cardiovascular events occurred in non-screened versus $2(0.41 \%)$ in screened patients $(n=1944$, Chi2 test, $p=0.14)$. There was no difference in the occurrence of CV events or major fractures between the 2 groups. In multivariate analysis, screening was associated with a $49 \%(0.51$ [0.41-0.64]) reduction in "all causes" hospitalization and a $27 \%(0,73$ [0.58-0.91]) decrease in "non-IRD" hospitalization, with no difference for CV or fracture cardiological events. The risk factors associated with "non-IRD" hospitalization were: history of "non-IRD" hospitalization in the previous year (2.26 [1.63-3.13]), IV bDMARDs (1.69 [1, 14-2.53]) and age> 70 years (1.44 [1.02-2.03] vs <50 years). Hospitalization in the previous year for "all causes" or "non-IRD" was associated with 